\title{
Capteur Brillouin réparti à fibre optique à haute résolution et longue portée
}

\author{
Thibaut Sylvestre ${ }^{1}$, Min Won Lee ${ }^{1}$, Duc Minh Nguyen ${ }^{1}$, \\ Birgit Stiller $^{1}$, Jean-Charles Beugnot ${ }^{1}$, Hervé Maillotte ${ }^{1}$, \\ Jérôme Hauden ${ }^{2}$, Luc Thévenaz ${ }^{3}$
}

1. Institut FEMTO-ST, Département d'Optique, Université de Franche-Comté, CNRS UMR $n^{\circ} 6174, F-25030$ Besançon

thibaut.sylvestre@univ-fcomte.fr

2. Photline Technologies, 25001 Besançon, France

Jerome.hauden@photline.com

3. Ecole Polytechnique Fédérale de Lausanne (EPFL), CH-1015 Lausanne, Suisse

luc.thevenaz@epfl.ch

RÉSUMÉ. Les capteurs répartis à fibre optique utilisant la diffusion Brillouin stimulée présentent un grand intérêt pour le contrôle permanent de l'intégrité et de la sécurité des systèmes et structures dans le génie civil ou l'industrie pétrolière. Ces capteurs permettent de mesurer avec précision des contraintes ou des variations de température le long des fibres optiques avec une résolution longitudinale de l'ordre du mètre sur plusieurs dizaines de kilomètres. Dans cet article, nous présentons deux nouveaux capteurs Brillouin répartis basés sur des formats avancés de modulation numérique empruntés aux systèmes modernes de télécommunications à haut débit. Nous montrons que ces nouvelles techniques de modulation permettent d'atteindre des résolutions spatiales centimétriques inférieures à la durée de vie des phonons acoustiques, et des distances d'interrogations allant jusqu'à cent kilomètres en insérant des amplificateurs optiques. Nous présentons également des cartographies des fibres optiques à cristal photonique à très haute résolution.

ABSTRACT. Brillouin-based distributed optical fiber sensors have been the subject of intense research in recent years because they offer a unique solution for continuous, real-time monitoring in civil engineering and petroleum industry. These sensors provide strain or temperature measurements with meter spatial resolution over several tens of kilometers. In this work we demonstrate two new Brillouin fiber sensors with enhanced performances based on advanced modulation formats from high-speed lightwave communications systems. We first report a Brillouin distributed sensor with enhanced centimeter resolution using a digital phase-shift keying technique. The second one uses a quadrature phase-shift keying modulator as a single-sideband modulator to balance the pump depletion and the fiber loss by the Brillouin gain. Combined with a specially-designed in-line bidirectional Erbium-doped fiber

Instrumentation, Mesure, Métrologie $-\mathrm{n}^{\circ} 1-2 / 2013,31-45$ 
amplifier, we demonstrate that this technique allows for the achievement of long-range distributed sensing over $100 \mathrm{~km}$.

MOTS-CLÉS : capteur à fibre optique, diffusion Brillouin.

KEYWORDS: distributed fiber sensors, Brillouin optical time domain analysis.

DOI:10.3166/I2M.13.1-2.31-45 (c) 2013 Lavoisier

\section{Introduction}

Les capteurs répartis à fibre optique présentent un grand intérêt pour le contrôle de l'intégrité et de la sécurité des systèmes et structures dans le génie civil ou l'industrie pétrolière (Omnisens, 2012). Ces capteurs à fibre permettent en effet de surmonter les limitations intrinsèques des capteurs traditionnels tels que les thermocouples ou les jauges de contraintes, tout en offrant des solutions de contrôle et de surveillance dans des environnements parfois inaccessibles et inhospitaliers. La fibre optique est un composant passif, flexible et insensible aux interférences électromagnétiques, ce qui en fait un candidat particulièrement intéressant pour jouer le rôle de capteur réparti lorsqu'elle est intégrée dans des structures civiles, des canalisations, ou des câbles. Une seule et unique fibre optique peut remplacer un grand nombre de capteurs ponctuels, offrant une réduction significative de l'installation, de l'étalonnage, et des coûts de maintenance.

Parmi les différentes technologies disponibles, les capteurs répartis à fibre optique utilisant la diffusion Brillouin dans le domaine temporel (BOTDA, pour Brillouin optical time domain analysis) permettent de mesurer avec une grande sensibilité des contraintes et des variations de température le long des fibres optiques sur plusieurs dizaines de kilomètres. Deux facteurs importants limitent cependant la résolution à un mètre et la distance d'interrogation à $50 \mathrm{~km}$ (Thévenaz et al., 2011). Il s'agit respectivement de la durée de l'impulsion pompe, limitée à $10 \mathrm{~ns}$ par le temps de vie des phonons acoustiques, et la déplétion de la pompe. En effet, la puissance de pompe diminue au cours de sa propagation en raison de l'absorption de la fibre et du transfert d'énergie vers la sonde Brillouin. Cet effet non local conduit généralement à une dégradation du rapport signal sur bruit, à une distorsion du spectre de gain Brillouin, et par conséquent à une estimation biaisée des coefficients de contrainte et de température. Plusieurs techniques ont été proposées récemment pour limiter ces deux effets afin d'augmenter les performances des capteurs Brillouin (Li et al. 2008 ; Soto et al., 2011 ; Bernini et al., 2011). La résolution peut être nettement améliorée par des méthodes différentielles utilisant plusieurs impulsions de profils ou de durées différentes (Brown et al., 2007 ; Li et al., 2008). Une alternative plus récente consiste à utiliser des sauts de phase très courts dans l'impulsion pompe qui vont bloquer subitement l'interaction acousto-optique et générer ce que l'on appelle des échos Brillouin (Thévenaz, 2010 ; Foaleng et al., 2011 ; Lee et al., 2012). La résolution spatiale peut ainsi dépasser la limite physique imposée par le temps de vie des phonons acoustiques et atteindre quelques centimètres (Thévenaz, 2010). Par ailleurs, la portée des capteurs Brillouin a été récemment étendue à $100 \mathrm{~km}$ en utilisant l'amplification 
Raman distribuée le long de fibres (Angulo-Vinuesa et al., 2011), $120 \mathrm{~km}$ à l'aide de la technique de codage des impulsions optiques (Bernini et al., 2011), et très récemment à $150 \mathrm{~km}$ en combinant différentes fibres optiques à l'amplification par fibre dopée à l'Erbium (Dong et al., 2012).

Dans cet article, nous présentons deux nouveaux capteurs répartis à fibre optique basés sur des formats avancés de modulation numérique, empruntés aux systèmes modernes de communications optiques cohérentes à très haut débit. Le premier capteur utilise une modulation digitale par déplacement de phase appelée DPSK (Differential Phase-Shift Keying). Avec cette technique, une modulation de phase de $\pi$ radians est obtenue par commutation positive/négative de la tension appliquée directement à un modulateur d'intensité autour de son point minimum, ce qui permet d'atteindre une résolution spatiale de $5 \mathrm{~cm}$ par la méthode différentielle des échos Brillouin (Lee et al., 2012). Le second système emploie un modulateur à deux interféromètres de MachZehnder parallèles de type I/Q (In-phase and Quadrature-phase) opérant dans un régime à bande latérale unique tout en supprimant efficacement la porteuse (Stiller et al., 2012 ; Nguyen et al., 2013). Combinée à un amplificateur optique bidirectionnel, cette technique de modulation permet une amélioration significative de la sensibilité du capteur et une distance d'interrogation atteignant $100 \mathrm{~km}$. Cet article résume en outre les travaux de recherche menés à l'Institut FEMTO-ST à Besançon dans le cadre d'un projet Franco-Suisse INTERREG IV en collaboration avec l'École Polytechnique Fédérale de Lausanne (EPFL) et les sociétés industrielles Photline Technologies, Omnisens, et Silitec fibers.

\section{Principe de fonctionnement}

Nos capteurs utilisent la rétrodiffusion Brillouin stimulée (SBS) d'une fibre optique qui se manifeste par une diffusion inélastique de la lumière induite par l'excitation d'ondes acoustiques et l'électrostriction (Kobyakov et al., 2010). Leur principe de fonctionnement est représenté sur la figure 1. Une impulsion lumineuse intense est injectée à une extrémité de la fibre, tandis qu'une sonde continue, décalée de la fréquence Brillouin à l'aide d'un modulateur électro-optique, est envoyée par l'autre extrémité de la fibre (Bao et al., 1993a, b; 1994 ; Nicklès et al, 1996 ; Horiguchi et al., 1995). Dans cette configuration, l'impulsion pompe transfère de l'énergie sur la sonde par le gain Brillouin suivant la relation de conservation suivante : $\boldsymbol{h} \boldsymbol{v}_{\boldsymbol{P}}=\boldsymbol{h} \boldsymbol{v}_{\boldsymbol{S}}+\boldsymbol{h} \boldsymbol{v}_{\boldsymbol{A}}$, où $\boldsymbol{v}_{\boldsymbol{A}}$ est la fréquence Brillouin du phonon acoustique. Comme la fréquence Brillouin d'une fibre optique dépend de la contrainte ou de la température appliquée sur celle-ci, le décalage de fréquence mesuré permet de rendre compte localement de la température ou de la contrainte, selon l'équation suivante :

$$
v_{A}(\varepsilon, T)=v_{A}(0,0)+C_{1} \times \varepsilon+C_{2} \times T
$$

où $\varepsilon$ et $\mathrm{T}$ sont la contrainte et la température. $C_{1}$ et $C_{2}$ sont les coefficients de contrainte et de température, qui sont de l'ordre de $5 \mathrm{MHz} / \mathbf{1 0 0} \boldsymbol{\mu} \varepsilon$ et $1,2 \mathrm{MHz} /{ }^{\circ} \mathrm{C}$ pour des fibres monomodes standard à la longueur d'onde de $1550 \mathrm{~nm}$. $\boldsymbol{v}_{\boldsymbol{A}}=$ $\mathbf{2} \boldsymbol{n}_{\text {eff }} \boldsymbol{V}_{\boldsymbol{a}} / \boldsymbol{\lambda}$ est la fréquence Brillouin de la fibre optique, avec $n_{\text {eff }}$ l'indice effectif 
du mode optique et $\lambda$ la longueur d'onde de la sonde. $V_{a}$ est la vitesse effective de l'onde acoustique longitudinale se propageant dans le cœur de la fibre optique. Elle est reliée à la répartition spatiale du mode acoustique et est proche de la vitesse acoustique dans la silice fondue $\left(V_{a}=5960 \mathrm{~m} . \mathrm{s}^{-1}\right)$. La résolution spatiale dépend de la durée de l'impulsion pompe de quelques dizaines de nanosecondes. Elle est limitée dans les systèmes classiques à 1 mètre par le temps de vie des phonons acoustiques responsables de la diffusion Brillouin (10 ns).

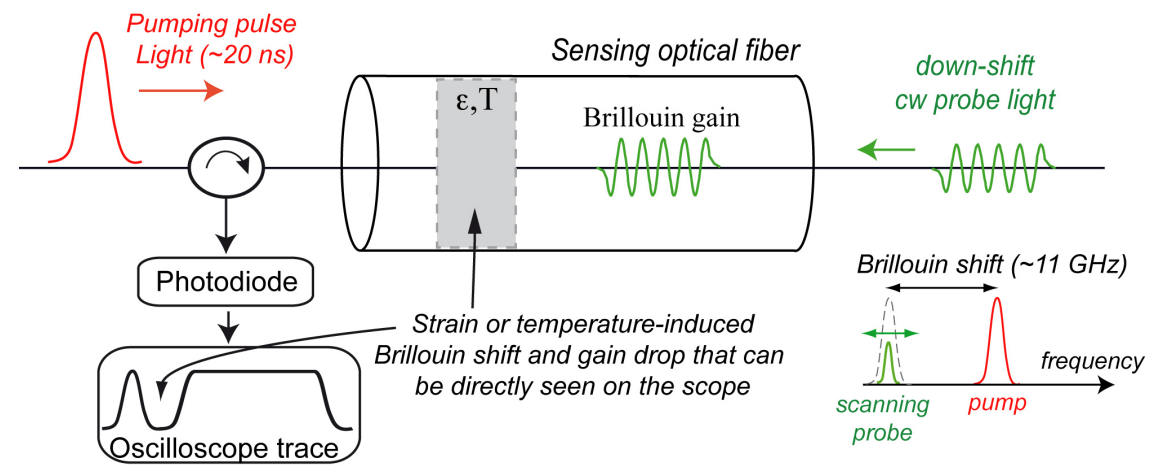

Figure 1. Schéma de principe de fonctionnement d'un capteur réparti à fibre optique basé sur la diffusion Brillouin

\section{Capteur Brillouin réparti à haute résolution}

Afin d'améliorer la résolution des capteurs Brillouin, une nouvelle technique de mesures distribuées dite «d'écho Brillouin»(BEDS : Brillouin Echo Distributed Sensing) a été développée initialement, par l'École Polytechnique Fédérale de Lausanne (Thévenaz, 2010). Cette technique est une méthode différentielle fondée sur l'utilisation d'une impulsion pompe modulée avec un saut abrupt de phase de $\pi$ radians. Son avantage est qu'elle permet d'améliorer d'un facteur dix la résolution spatiale longitudinale par rapport aux systèmes existants tout en procurant une bonne sensibilité. La technique des échos Brillouin nécessite cependant l'usage de deux modulateurs intégrés pour la pompe Brillouin: un modulateur Mach-Zehnder d'intensité formant une impulsion pompe à profil rectangulaire et un modulateur de phase permettant d'appliquer un saut de phase de $\pi$ radians à la fin de l'impulsion rectangulaire. Obtenir une résolution ultime nécessite alors de contrôler le décalage relatif des impulsions électriques de commande de chaque modulateur avec un haut degré de précision. Afin de simplifier le système expérimental et pouvoir obtenir plus aisément une résolution élevée, nous avons, en collaboration étroite avec l'École Polytechnique Fédérale de Lausanne (EPFL) et la société Photline Technologies, mis au point une nouvelle configuration reposant sur une technique dérivée des formats avancés de modulation. Ces nouveaux formats sont actuellement utilisés pour coder en 
phase les séquences de bits à très haut débit dans les transmissions numériques par fibre optique (Charlet, 2006 ; Lee et al., 2012 ; Stiller et al., 2012).

Dans cette méthode de codage en phase dite «DPSK » (Differential Phase-Shift Keying), une modulation de phase de $\pi$ radians est obtenue par commutation positive/négative de la tension demi-onde appliquée à un modulateur d'intensité. Ce saut de phase de $\pi$ radians va permettre d'interrompre subitement l'interaction Brillouin et l'onde acoustique va s'atténuer progressivement tout en renvoyant un écho sur la sonde. Cette modulation est représentée schématiquement sur la figure 2a. Dans le cadre de notre système, deux impulsions électriques, une positive et une négative, appliquées au même modulateur Mach-Zehnder (MZ), forment en sortie de celui-ci deux impulsions adjacentes déphasées de $\pi$ radians, la deuxième impulsion ultra-brève étant ajustée à une durée de 500 ps afin d'augmenter la résolution spatiale du capteur. Cette configuration est donc simplifiée et améliore le bilan de puissance optique du système de mesure tout en conservant l'efficacité propre à la méthode BEDS puisque la mise en forme de la pompe Brillouin ne nécessite plus qu'un unique modulateur d'intensité. Une résolution spatiale de $5 \mathrm{~cm}$ et une bonne sensibilité peuvent être aisément obtenues en comparant directement l'impulsion avec et sans saut de phase.

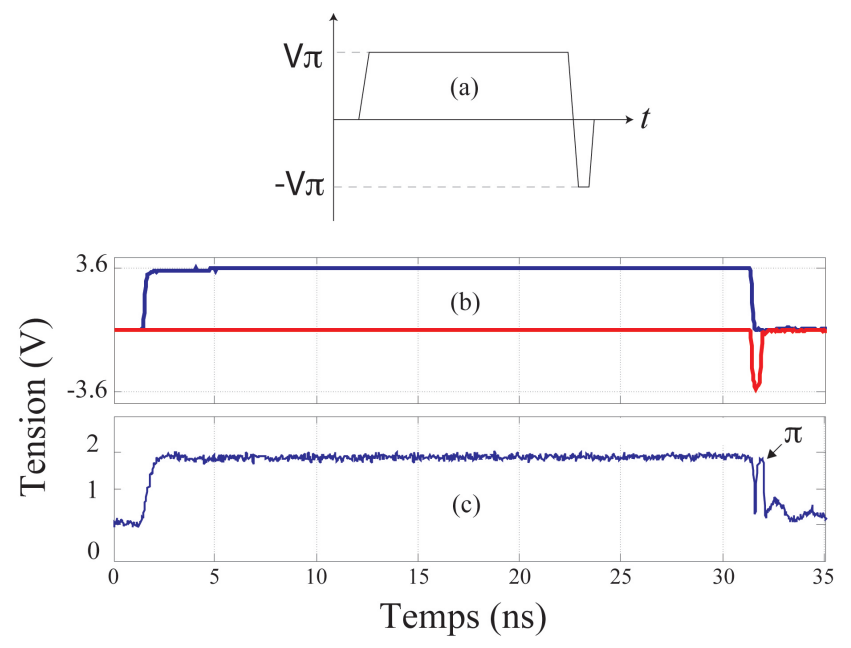

Figure 2. (a) Schéma de l'impulsion électrique positive/négative envoyée sur le modulateur Mach-Zehnder. (b) Impulsions électriques mesurées sur l'oscilloscope. (c) Intensité optique en sortie du modulateur. Les durées des impulsions d'intensité longue et de phase courte sont respectivement 30 ns et 500 ps

Cette impulsion d'intensité à double état de phase est ensuite utilisée dans le montage expérimental de notre capteur à fibre optique présenté sur la figure 3 . Le capteur utilise comme source lumineuse un laser à $1550 \mathrm{~nm}$ à contre-réaction distribuée (DFB). L'émission continue de ce laser est séparée en deux à l'aide d'un 
coupleur à fibre pour la pompe et la sonde. Sur le modulateur MZ (Photline MXPE series) de la pompe, un T-Bias RF est utilisé pour la combinaison d'impulsions positives et le DC. La tension DC est ajustée au point de fonctionnement minimal du Modulateur MZ. Les impulsions sont amplifiées par un amplificateur à fibre dopée Erbium (EDFA) avec une puissance crête de $1.3 \mathrm{~W}$. Puis la polarisation est brouillée de façon à moyenner le gain Brillouin sensible à la polarisation. La pompe est injectée dans la fibre optique monomode avec un circulateur. La sonde est décalée de la fréquence Brillouin autour de $11 \mathrm{GHz}$ par un deuxième modulateur d'intensité avec une suppression de porteuse de $38 \mathrm{~dB}$ en ajustant le DC. La puissance de sonde est réglée à $1 \mathrm{~mW}$ avec un autre EDFA. La sonde est injectée dans la fibre après un isolateur. Un réseau de Bragg fibré (FBG) est utilisé pour sélectionner la sonde en sortie de fibre. L'intensité de la sonde est finalement mesurée à l'aide d'un photodétecteur connecté à un oscilloscope numérique. En balayant la fréquence de la sonde autour de la fréquence Brillouin de la fibre, le spectre de gain Brillouin peut ainsi être cartographié en fonction de la fréquence.

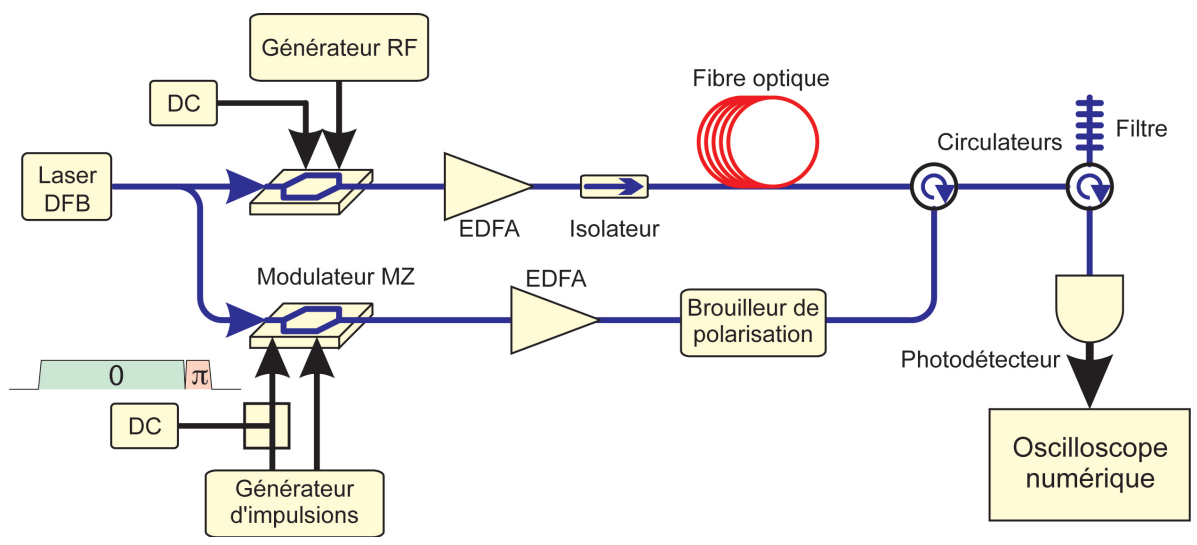

Figure 3. Montage expérimental du capteur Brillouin réparti à fibre optique à haute résolution spatiale $(5 \mathrm{~cm})$ basé sur les échos Brillouin. EDFA: amplificateur à fibre dopée Erbium

Afin de démontrer la performance de la technique développée dans ce travail, nous avons dans un premier temps cartographié une épissure entre deux fibres optiques de nature différente. De façon plus précise, nous avons soudé une fibre monomode standard (SMF) de $2 \mathrm{~m}$ de long à une fibre à grande ouverture numérique (HNA) de $1 \mathrm{~m}$. La zone d'épissure est protégée par une gaine thermorétractable de $5 \mathrm{~cm}$ qui exerce une pression sur ces deux fibres. La figure 4 présente la mesure distribuée du spectre de gain Brillouin obtenue avec notre système BEDS avec une impulsion de phase de durée $500 \mathrm{ps}$. La zone d'épissure de $5 \mathrm{~cm}$ entre les 2 fibres est clairement visible avec un décalage de fréquence associé de $10,55 \mathrm{GHz}$ induit par la gaine thermorétractable. Les fréquences Brillouin des fibres HNA et SMF sont mesurées respectivement à $10,67 \mathrm{GHz}$ et $10,85 \mathrm{GHz}$. 


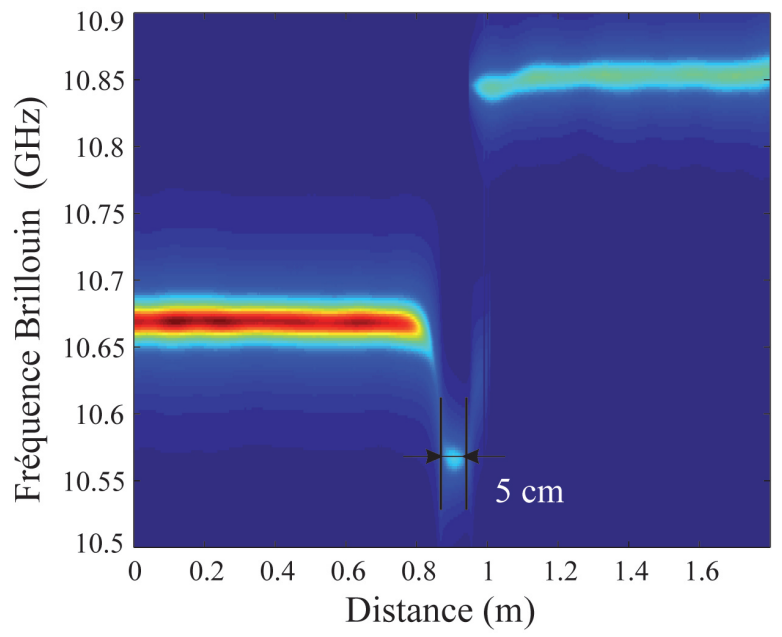

Figure 4. Cartographie d'une épissure entre deux fibres optiques de nature différente montrant l'effet de la gaine thermorétractable avec une résolution longitudinale de $5 \mathrm{~cm}$

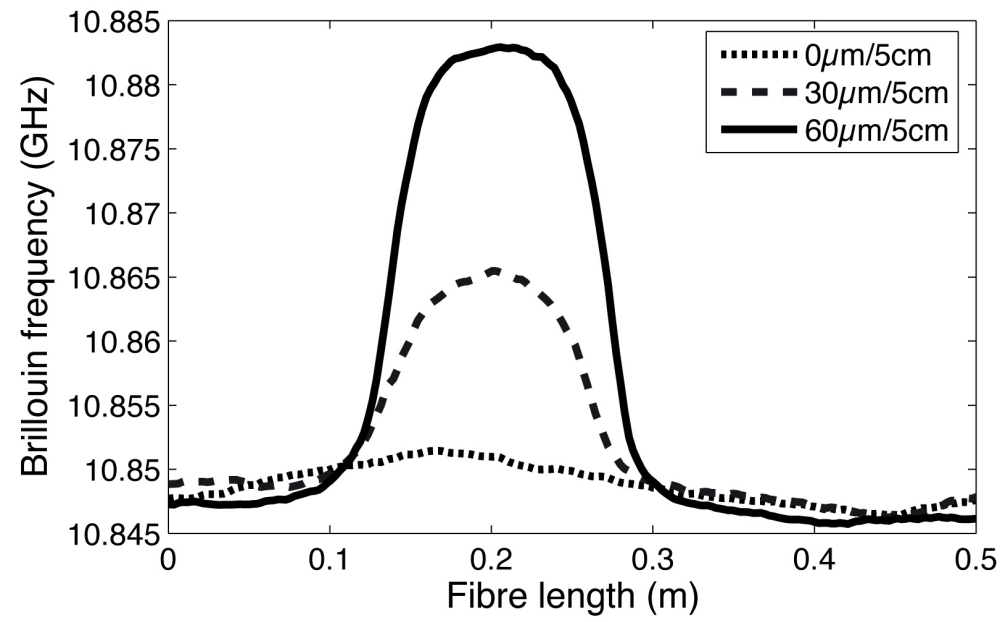

Figure 5. Décalage de la fréquence Brillouin d'une fibre optique monomode (SMF) de $260 \mathrm{~m}$ de long induit par élongations successives de 30 et $60 \mu \mathrm{m}$

d'une portion de $5 \mathrm{~cm}$

A partir de ce système, une plateforme de mesure d'élongations de fibres optiques a été mise en place. Une première campagne expérimentale de validation a été réalisée en utilisant dans un premier temps comme capteur, une fibre monomode 
standard $(\mathrm{SMF})$ dans le banc de mesure. Une résolution spatiale de $5 \mathrm{~cm}$ a là aussi pu être obtenue dans la mesure d'élongations localisées appliquées sur une portion de fibre de $260 \mathrm{~m}$, avec une résolution fréquentielle du déplacement du spectre de gain Brillouin de l'ordre du MHz. Les résultats sont présentés dans la figure 5. La sensibilité à la traction obtenue, de $3 \mathrm{MHz}$ pour $100 \mu \varepsilon$, est légèrement inférieure à la valeur nominale de $5 \mathrm{MHz}$ en raison du protocole expérimental d'élongation mis en œuvre. En remplaçant cette fois la fibre standard par une fibre optique microstructurée, des tests préliminaires effectués très récemment ont montré que ce type de fibre présente un décalage linéaire de son spectre Brillouin en fonction de l'étirement de l'ordre de $5 \mathrm{MHz}$ pour $100 \mu \varepsilon$, équivalent aux fibres standard, tout en procurant un accroissement significatif de sensibilité par le gain Brillouin élevé. Ces résultats préliminaires montrent tout le bien-fondé de l'emploi de fibres optiques microstructurées pour une application aux capteurs répartis.

\section{Capteur Brillouin réparti à grande sensibilité}

Un des principaux obstacles limitant la sensibilité des capteurs à fibre est la déplétion de la pompe. En effet, la puissance de l'impulsion pompe diminue au cours de sa propagation dans la fibre optique en raison de l'absorption de la fibre et du transfert d'énergie vers la sonde Brillouin. Cet effet de déplétion conduit généralement à une dégradation du rapport signal sur bruit et une baisse de la sensibilité du capteur le long de la fibre optique, et par conséquent à une estimation biaisée des paramètres environnementaux.

Dans cette section, nous présentons un capteur Brillouin réparti utilisant la modulation à bande latérale unique (BLU) anti-Stokes (Nguyen et al., 2013). Cette technique, basée sur la mesure des pertes anti-Stokes au lieu du gain Stokes, permet effectivement de compenser la déplétion de la pompe par transfert d'énergie de la bande latérale anti-Stokes et ainsi améliorer de façon significative la sensibilité du capteur. Cette modulation originale est effectuée à l'aide d'un modulateur à deux interféromètres de Mach-Zehnder (MZ) parallèles (modulateur I\&Q) qui ont été principalement développés pour les communications cohérentes de type QPSK (quadrature-phase shift keying). Notre technique permet de générer la bande latérale unique anti-Stokes avec une suppression de porteuse de $45 \mathrm{~dB}$ ainsi qu'un taux d'extinction de la bande latérale Stokes de $35 \mathrm{~dB}$ à $1535 \mathrm{~nm}$ (cf. figure 6). Nos résultats de mesures sur une distance de $50 \mathrm{~km}$ de fibre standard montrent une meilleure sensibilité du capteur aux déformations en comparaison avec le capteur conventionnel utilisant la modulation à deux bandes latérales symétriques.

Le schéma de principe de ce capteur Brillouin est présenté à la figure 6. Il reprend les principaux éléments du montage précédent (cf. figure 3), mis à part les techniques de modulation de la pompe et de la sonde. Une onde continue (CW) à $1550 \mathrm{~nm}$ provenant d'un autre laser DFB est divisée en deux par un coupleur à fibre. La pompe est ensuite modulée par un modulateur d'intensité pour générer des impulsions de durée $30 \mathrm{~ns}$ sur une période de $550 \mathrm{~ms}$. Les impulsions sont ensuite amplifiées avec un amplificateur à fibre dopée Erbium (EDFA) puis injectées dans le capteur à fibre via un circulateur optique. Pour la sonde, nous utilisons cette fois un modulateur I/Q pour 
générer une bande latérale unique anti-Stokes sans porteuse, comme le montre l'encart dans la figure 6. De façon plus précise, il s'agit d'un modulateur avec deux interféromètres de Mach-Zehnder parallèles nichés dans un troisième interféromètre de Mach-Zehnder (combineur). Grâce à une tension de polarisation DC, les deux interféromètres sont polarisés au minimum de leur transmission afin de supprimer la porteuse optique. Un signal RF modulé à la fréquence Brillouin autour de $11 \mathrm{GHz}$ est injecté dans les deux ports du modulateur avec un décalage de phase de $\pi / 2$. Une tension DC appliquée au combineur, permet de régler la phase relative des signaux de sortie des interféromètres Mach-Zehnder. Lorsqu'un déphasage électrique de $\pi / 2$ est introduit à l'entrée, et un déphasage optique de $\mp \pi / 2$ est introduit par le combineur, on atteint un fonctionnement BLU haute ou basse sans porteuse. Le choix de la bande latérale unique « haute » ou « basse » ne dépend que du signe du déphasage optique de $\pi / 2$ (Shimotsu et al., 2001). On peut alors choisir une BLU anti-Stokes ou Stokes en ajustant simplement la tension DC appliquée sur le combineur. Cette sonde est ensuite injectée dans la fibre optique après un isolateur. A la sortie de la fibre, la sonde antiStokes Brillouin est extraite par le circulateur puis analysée simultanément par un oscilloscope et un analyseur de spectre optique. Il est également important de souligner que cette méthode permet de s'affranchir du filtre passe-bande de sortie puisque seule la bande anti-Stokes est présente.

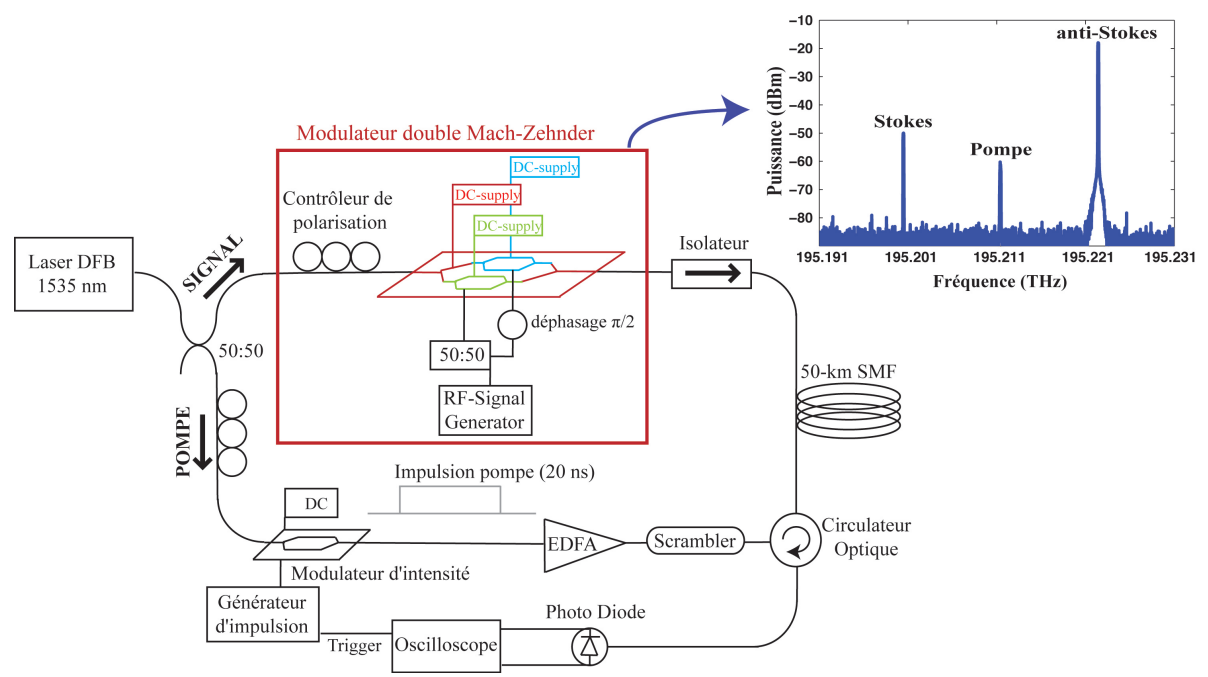

Figure 6. Schéma du capteur Brillouin réparti à fibre optique à grande sensibilité utilisant la technique de modulation à bande latérale unique (BLU) anti-Stokes.

$L$ 'encart représente le spectre de la sonde en sortie du modulateur

En balayant la fréquence de la sonde autour de la fréquence Brillouin de la fibre, on parvient ainsi à cartographier le spectre de gain Brillouin en fonction de la distance parcourue dans la fibre, comme le montre la figure $7 \mathrm{a}$. Toutes les variations 
de fréquence Brillouin induites par des déformations ou des changements de température peuvent ainsi être détectées le long de la fibre.

Pour détecter ces variations, nous avons étiré l'extrémité de la fibre de $66 \mathrm{~mm}$ pour une distance de $24 \mathrm{~m}$. La figure 7a montre la variation de la fréquence Brillouin à l'extrémité de la fibre induite par cette élongation. On mesure ainsi un décalage de $35 \mathrm{MHz}$ de la fréquence Brillouin, comme le montre la figure 7a. Le décalage de la fréquence Brillouin en fonction de l'élongation appliquée sur la fibre est illustré sur la figure $7 \mathrm{~b}$.
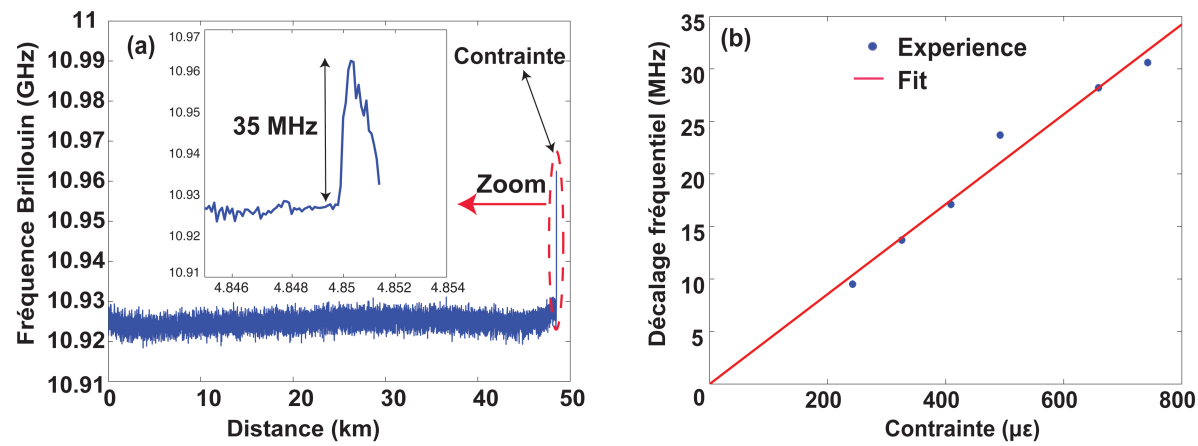

Figure 7. Détection d'une contrainte appliquée en fin de fibre.

(a) Fréquence Brillouin avec une élongation de $2750 \mathrm{~mm} / \mathrm{m}$ sur $24 \mathrm{~m}$ en fin de fibre. (b) Mesure du coefficient de contrainte : décalage de la fréquence Brillouin en fonction de l'élongation de la fibre

On obtient ainsi le coefficient de déformation de $4,8 \mathrm{MHz} / 100 \mu \varepsilon$, en très bon accord avec les valeurs reportées dans la littérature $\left(\mathrm{C}_{1}=5 \mathrm{MHz} / 100 \mu \varepsilon\right)$. Ces résultats montrent qu'il n'y a manifestement pas d'erreur introduite par une éventuelle déplétion de la pompe dans le cas de la sonde anti-Stokes.

Nous avons ensuite comparé la technique de modulation BLU anti-Stokes avec la méthode standard à deux bandes latérales symétriques (DSB). Les résultats sont présentés sur la figure 8 . En particulier, la figure $8 \mathrm{c}$ montre une sensibilité augmentée d'un facteur 5 en fin de fibre dans le cas de la modulation BLU antiStokes dans les mêmes conditions de fonctionnement. La figure 8c fait apparaître en particulier un gain négatif $(-1<$ gain $<0)$ pour le régime BLU anti-Stokes car nous mesurons dans ces conditions les pertes Brillouin plutôt que le gain sur la sonde Stokes dans le régime DBL. La distance d'interrogation du capteur utilisant la modulation standard DBL est quant à elle limitée à environ $40 \mathrm{~km}$ à cause de la forte atténuation de la pompe. La figure 8d compare les spectres de gain Stokes et de perte anti-Stokes en fin de fibre optique. La courbe en pointillés montre clairement un dédoublement du spectre de gain Brillouin pour la modulation à deux bandes latérales qui témoigne de l'impact de la déplétion de la pompe, tandis que le spectre de perte anti-Stokes reste symétrique et centré sur la fréquence Brillouin de la fibre. 

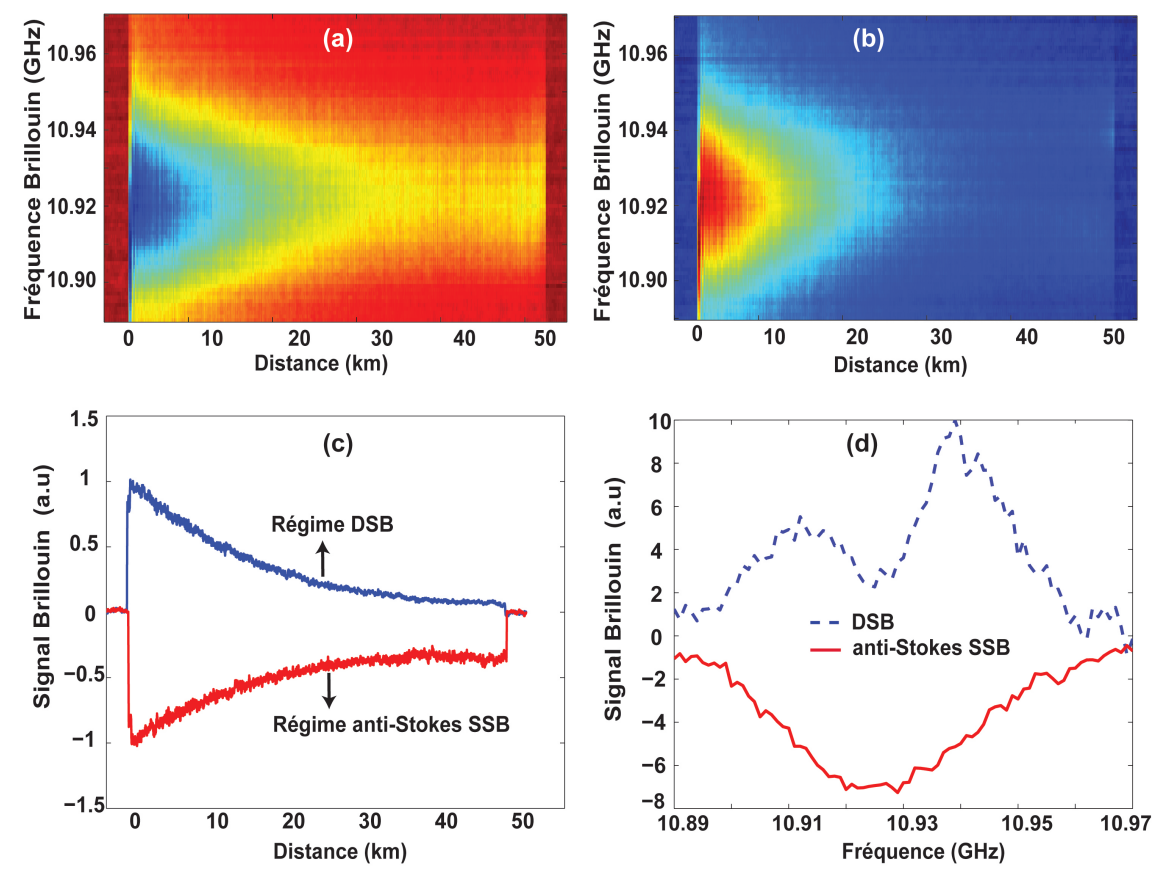

Figure 8. Intensité de la sonde Brillouin en fonction de la fréquence de balayage et de la distance dans la fibre optique et comparaison directe des techniques de modulation à bande latérale unique anti-Stokes (a) et à deux bandes latérales (b). (c) Comparaison directe de la sonde Stokes dans le régime DSB et de la sonde antiStokes dans le régime SSB. (d) Comparaison des spectres de gain Stokes et de perte anti-Stokes en fin de fibre optique

\section{Capteur Brillouin réparti à longue portée}

A partir de la technique développée précédemment, nous avons réalisé un capteur réparti à longue portée $(100 \mathrm{~km})$ en combinant la technique de modulation à bande latérale unique à l'amplification optique bidirectionnelle. A l'aide de la technique BLU anti-Stokes, nous pouvons désormais réduire la déplétion de la pompe sans avoir recours à la technique de multiplexage en fréquence Brillouin avec l'utilisation de différentes fibres (Dong et al., 2012). Le montage expérimental est schématisé sur la figure 8. Il compte désormais deux bobines de $50 \mathrm{~km}$ de fibres SMF qui sont reliées entre elles par un amplificateur à fibre optique dopée à l'Erbium. Cet amplificateur optique bidirectionnel a été spécialement conçu par Manlight à Lannion pour amplifier simultanément la pompe impulsionnelle dans un sens de propagation et la sonde continue dans le sens contra-propagatif. Il est composé de deux fibres Erbium pompées indépendamment l'une de l'autre et reliées par deux circulateurs optiques à chaque extrémité. Les deux circulateurs permettent d'injecter la pompe et la sonde 
dans chaque fibre Erbium sans qu'ils n'interagissent entre eux. Différents modes d'amplification peuvent être appliqués indépendamment pour optimiser le gain, la puissance de sortie, ainsi que les facteurs de bruit de la pompe ou de la sonde. La période des impulsions émises par la pompe a été ajustée à $1,15 \mu$ s pour assurer une seule et unique impulsion sur la longueur de propagation dans les deux fibres. Sa durée a été fixée à $70 \mathrm{~ns}$ ce qui correspond à une résolution spatiale de $7 \mathrm{~m}$.

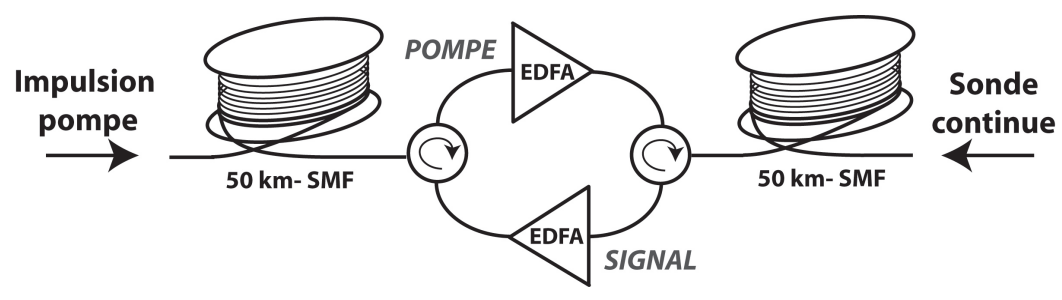

Figure 9. Schéma de principe de l'amplification optique bidirectionnelle en milieu de ligne permettant d'augmenter la portée des capteurs répartis à $100 \mathrm{~km}$

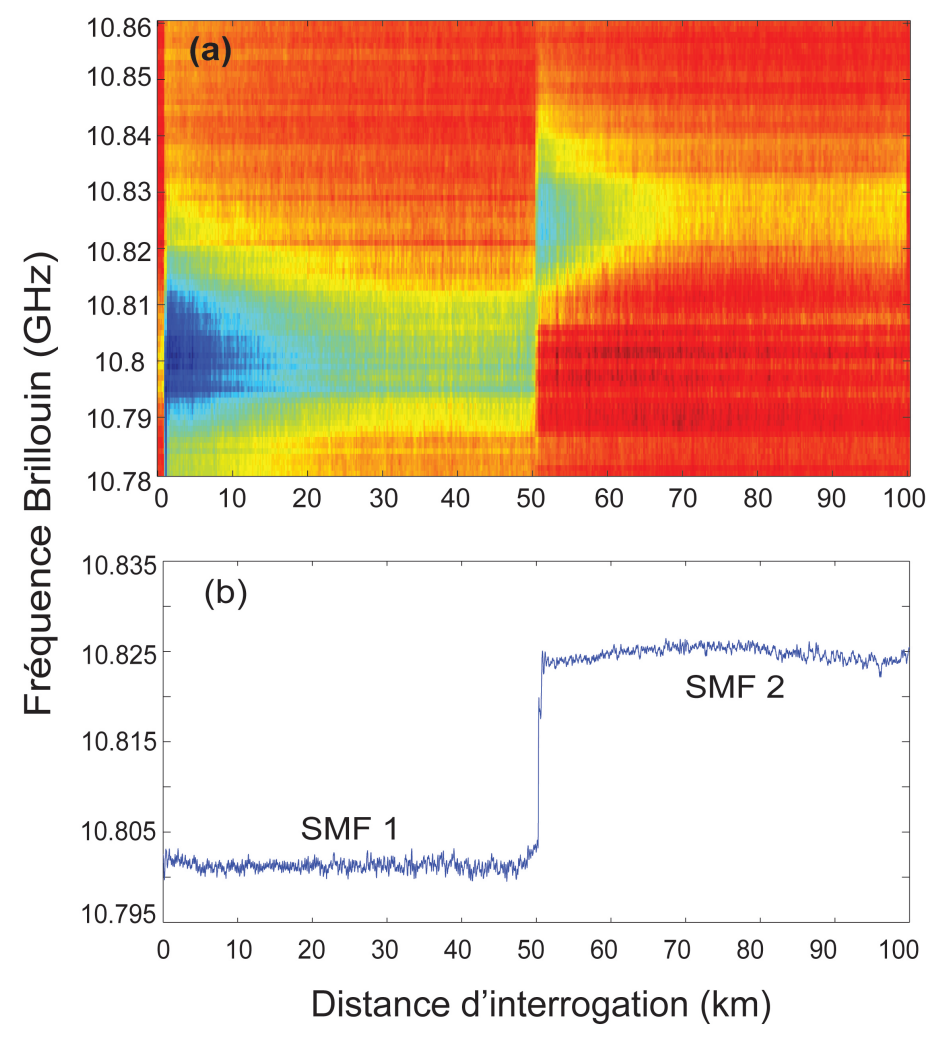

Figure 10. (a) Intensité en fausse couleur de la sonde Brillouin en fonction de la fréquence de balayage et de la distance dans les deux fibres optiques de $50 \mathrm{~km}$.

(b) Fréquence Brillouin le long des 2 fibres 
La figure 10a présente une cartographie en fausse couleur du spectre de gain Brillouin réparti sur les deux fibres optiques concaténées. Cette figure montre clairement l'efficacité de l'amplificateur bidirectionnel à régénérer les signaux pompe et sonde. La figure 10b montre les fréquences Brillouin réparties sur les deux fibres légèrement décalées à $10,801 \mathrm{GHz}$ et $10,824 \mathrm{GHz}$. Ce décalage est probablement dû au processus d'étirage des fibres optiques. Nous avons ensuite mesuré le coefficient de contrainte en étirant la fibre sur la fin des $100 \mathrm{~km}$. Comme précédemment, nous avons étiré l'extrémité de la fibre sur une distance de $24 \mathrm{~m}$. La figure 11 montre le décalage de la fréquence Brillouin en fonction du facteur d'élongation de la fibre. Nous avons mesuré par un ajustement linéaire un coefficient de contrainte de $5,3 \pm 0,5 \mathrm{MHz} / 100 \mu \varepsilon$, ce qui est en très bon accord avec les valeurs standard, confirmant ainsi l'efficacité de l'amplificateur EDFA pour supprimer la déplétion de pompe.

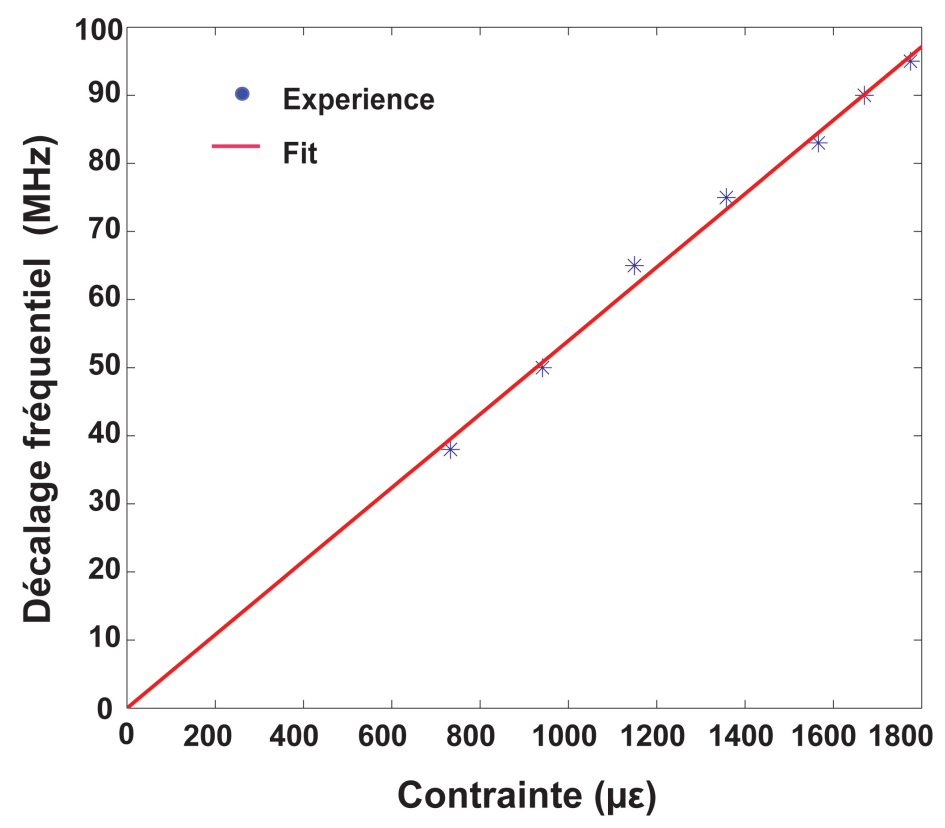

Figure 11. Mesure du coefficient de contrainte. Décalage de la fréquence Brillouin en fonction de l'élongation de la fibre appliquée à la fin des $100 \mathrm{~km}$ de propagation

\section{Conclusion}

En conclusion, nous avons démontré dans ce travail que les nouveaux formats avancés de modulation numérique, utilisés principalement pour les télécommunications optiques, présentent également un grand intérêt pour les capteurs répartis à fibre optique. De façon plus précise, ces nouvelles techniques de 
modulation permettent d'augmenter la résolution spatiale ou la sensibilité des capteurs répartis à fibre optique pour des applications spécifiques au contrôle de l'intégrité et de la sécurité des systèmes et structures dans le génie civil ou l'industrie pétrolière. Nous avons dans un premier temps démontré une amélioration d'un facteur $5 \mathrm{du}$ signal rapport sur bruit et une portée à $50 \mathrm{~km}$ en utilisant la technique de modulation à bande latérale unique anti-Stokes. Puis nous avons étendu la distance d'interrogation du capteur réparti à $100 \mathrm{~km}$ en combinant cette technique de modulation avec un amplificateur bidirectionnel en ligne.

\section{Bibliographie}

Angulo-Vinuesa X., Martin-Lopez S., Nuno J., Corredera P., Ania-Castanon J.-D., Thévenaz L., Gonzalez-Herraez M. (2011). Hot spot detection over $100 \mathrm{~km}$ with $2 \mathrm{~m}$ resolution in a Raman-assisted Brillouin distributed sensor. Proceedings SPIE, 7753, 775309.

Bao X., Webb D. J. Jackson D. A. (1994). Combined distributed temperature and strain sensor based on Brillouin loss in an optical fiber. Optics Letters 19, p. 141-143.

Bao X., Webb D. J., Jackson D. A. (1993a). 32-km distributed temperature sensor based on Brillouin loss in an optical fiber. Optics Letters, vol. 18, $\mathrm{n}^{\circ} 18$, p. 1561-1563.

Bao X., Webb D. J., Jackson D. A. (1993b). 22-km distributed temperature sensor using Brillouin gain in an optical fiber. Optics Letters, vol. 18, n 7, p. 552-554.

Bernini R., Minardo A., Zeni L. (2011). Long-range distributed Brillouin fiber sensors by use of an unbalanced double sideband probe. Optics Express, vol.19, n 24, p. 23845-23856.

Brown A. W., Colpitts B. G., Brown K. (2007). Dark-pulse Brillouin optical time-domain sensor with 20-mm spatial resolution. J. Lightwave Technol., vol. 25, p. 381-386.

Charlet G. (2006). Progress in optical modulation formats for high-bit rate WDM transmissions. IEEE J. Quantum Electron. 12, p. 469-483.

Domingo J. M. S., Pelayo J., Villuendas F., Heras C. D., Pellejer E. (2005). Very high resolution optical spectrometry by stimulated Brillouin scattering. IEEE Photonic Technology Letters, vol. 17, $\mathrm{n}^{\circ}$ 4, p. 855-857.

Dong Y., Chen Li., Bao X. (2012). Extending the Sensing Range of Brillouin Optical TimeDomain Analysis Combining Frequency-Division Multiplexing and In-Line EDFAs. J. Lightwave Technol. 30, p. 1161-1167.

Foaleng S. M., Tur M., Beugnot J.-C., Thévenaz L. (2011). High spatial and spectral resolution long-range sensing using Brillouin echoes. J. Lightwave Technol., vol. 28, p. 2993-3003.

Horiguchi T., Shimizu K., Kurashima T., Tateda M., Koyamada Y. (1995). Development of a distributed sensing technique using Brillouin scattering. J. Lightw. Technol. 13, p. 12961302 .

Horiguchi T., Tateda M. (1989). Optical-fiber-attenuation investigation using stimulated Brillouin scattering between a pulse and a continuous wave. Optics Letters, vol. $14, \mathrm{n}^{\circ} 8$, p. $408-410$. 
Kobyakov A., Sauer M., Chowdhury D. (2010). Stimulated Brillouin scattering in optical fibers. Adv. Opt. Photon. 2, p. 1-59.

Kurashima T., Horiguchi T., Tateda M. (1990). Distributed-temperature sensing using stimulated Brillouin scattering in optical silica fibers. Optics Letters, vol. 15, p. 10381040 .

Lee M. W., Stiller B., Hauden J., Maillotte H., Roch C., Thévenaz L., Sylvestre T. (2012). Differential phase-shift keying technique-based Brillouin echo-distributed sensing. IEEE Photonic Technology Letters, vol. 24, n 1, p. 79-81.

Li W., Bao X., Li Y., Chen L. (2008). Differential pulse-width pair BOTDA for high spatial resolution sensing. Optics Express, vol. 16, p. 21616-21625.

Loayssa A., Hernandez R., Benito D., Galech S. (2004). Characterization of stimulated Brillouin scattering spectra by use of optical single-sideband modulation. Optics Letters vol. $29, \mathrm{n}^{\circ} 6$, p. 638-640.

Nguyen D. M., Stiller B., Lee M. W., Beugnot J.-C, Mottet A., Hauden J., Maillotte H., Sylvestre T. (January, 2013). Distributed Brillouin Fiber Sensor with Enhanced Sensitivity based on Anti-Stokes Single-Sideband Suppressed-Carrier Modulation. IEEE Photon. Technol. Lett., vol. 25, n 1, p. 94-96.

Niklès M., Thévenaz L., Robert P. A. (1996). Simple distributed fiber sensor based on Brillouin gain spectrum analysis. Optics Letters, vol. 21, n 10, p. 758-760.

Omnisens (2012), Securing asset integrity with Omnisens, an overview. http://www. omnisens.ch/

Shimotsu S., Oikawa S., Saitou T., Mitsugi N., Kubodera K., Kawanishi T., Izutsu M. (2001) Single Side-Band Modulation Performance of a LiNbO3 Integrated Modulator Consisting of Four-Phase Modulator Waveguides. IEEE Photonic Technology Letters, vol. 13, $\mathrm{n}^{\circ} 4$, p. $364-366$.

Song K.-Y., Hotate K. (2006). Enlargement of measurement range in a Brillouin optical correlation domain analysis system using double lock-in amplifiers and a single-sideband modulator. IEEE Photonic Technology Letters, vol. 18, n 3, p. 499-501.

Song K. Y., Yoon H. J. (2010). High-resolution Brillouin optical time domain analysis based on Brillouin dynamic grating. Optics Letters, vol. 35, n 1, p. 52-54.

Soto M. A., Bolognini G., Pasquale F. D. (2011). Long-range simplex-coded BOTDA sensor over $120-\mathrm{km}$ distance employing optical preamplification. Optics Letters, vol. 36, $\mathrm{n}^{\circ} 2$, p. $232-234$.

Stiller B., Lee M. W., Nguyen D. M., Hauden J., Mottet A., Maillotte H., Sylvestre T. (2012). Fiber optic Brillouin distributed sensing using phase-shift keying modulation techniques. Proceedings of SPIE 8439, SPIE Photonics Europe, Brussel.

Thévenaz L. (2010). Brillouin distributed time-domain sensing in optical fibers: state of the art and perspectives. Frontiers of Optoelectronics in China, vol. 3, p. 13-21.

Thévenaz L., Mafang S. F., Lin J. (2011). Depletion in a distributed Brillouin fiber sensor: Practical limitation and strategy to avoid it. Proc. $21^{\text {st }}$ Int. Conf. Opt. Fiber Sensors. 